\title{
Review
}

\section{Learning During Stressful Times}

\author{
Tracey J. Shors \\ Department of Psychology, Center for Collaborative Neuroscience, Rutgers University, Piscataway, New Jersey 08854, USA
}

\begin{abstract}
Stressful life events can have profound effects on our cognitive and motor abilities, from those that could be construed as adaptive to those not so. In this review, I discuss the general notion that acute stressful experience necessarily impairs our abilities to learn and remember. The effects of stress on operant conditioning, that is, learned helplessness, as well as those on classical conditioning procedures are discussed in the context of performance and adaptation. Studies indicating sex differences in learning during stressful times are discussed, as are those attributing different responses to the existence of multiple memory systems and nonlinear relationships. The intent of this review is to highlight the apparent plasticity of the stress response, how it might have evolved to affect both performance and learning processes, and the potential problems with interpreting stress effects on learning as either good or bad. An appreciation for its plasticity may provide new avenues for investigating its underlying neuronal mechanisms.
\end{abstract}

It must be that stress impairs learning—or does it? In this review, I describe some basic trends in studies that have demonstrated a relationship between stress and learning. Then I present exceptions, the numbers of which are significant. This is not an attempt to review the literature on stress and learning, which is immense, or to review the neurobiological substrates that may underlie them, which is nearly as immense. Rather, my intention is to use a few examples from the literature, primarily from our laboratory and those associated with "learned helplessness" phenomena, to illustrate the precarious nature of the relationship between stress and learning, and what that nature implies for identifying neurobiological substrates and mechanisms. More generally, the intention of this review is to pose alternatives to the simple idea that stress impairs memory processes, highlighting the evidence that stressful experience has multiple, and sometimes, even opposite effects on our abilities to learn and remember.

Stress and Operant Conditioning (Learning Helplessness) The most well-known effect of stress on learning is learned helplessness. In the 1960s, a group of psychologists working at the University of Pennsylvania noticed that animals exposed to brief inescapable shocks were markedly impaired in their subsequent ability to learn a new task (Overmier and Seligman 1967; Seligman and Maier 1967). Those that were exposed to the same amount of shock, but had control over it, were not impaired. These experiments provided evidence that a psychological variable such as uncontrollability could later retard an animal's capacity to respond when control was possible. Performance was most often assessed during operant conditioning, a training situation in which animals elicit overt motor responses in order to be reinforced (Glazer and Weiss 1976; Maier and Jackson 1979; Minor et al. 1988, 1991; Shors and Dryver 1992). Animals exposed to the inescapable stress expressed a number of other symptoms such as sleep and eating disturbances, ulcers, and decreases in immune status. Because it appeared as if the animals had given up, the phenomenon was promoted as an animal model for depression in humans (Seligman 1975), the rationale being that exposure to uncontrollable and stressful life events leads to a feeling of loss of control, which ultimately leads to depressivelike behavior.

E-MAIL shors@rci.rutgers.edu; FAX (732) 445-2263.

Article and publication are at http://www.learnmem.org/cgi/doi/10.1101/ Im.66604.
Although generally accepted today, the helplessness phenomenon was historically a controversial observation-or at least the interpretations of it were. There were two general theories that emerged during the height of the controversy, one being cognitive in nature and the other performance based (Maier and Jackson 1979). The cognitive interpretation asserted that during exposure to the inescapable shocks, the animal learns that the shock and its response are not contingent and is impaired thereafter in forming relevant contingencies. The other prevailing theory was that the performance decrement reflects a decrease in activity, which results either from associating the shock with an inactive response, or is simply a product of neurotransmitter depletion (Glazer and Weiss 1976; Anisman et al. 1978; Sherman and Petty 1980). There were a number of attempts to dissociate the effects of stress on activity from those on performance, and some results suggested that the helplessness effect was one of learning, whereas others did not. In one study, rats were exposed to an inescapable stressor of intermittent shocks and tested later in an avoidance-escape task in which the response required minimal movement (a nose-poke response). Animals exposed to the stressor actually performed better than the unstressed controls (Glazer and Weiss 1976). In another study, the effect of stress on choice in a Y-maze task was examined. Along with deficits in activity, there was an increase in the number of incorrect choices made by the stressed animals (Jackson et al. 1980). Subsequent experiments suggested that the decreased performance was a result of deficits in selective attention rather than learning, per se (Minor et al. 1984). There were many such studies conducted with similar conflicting results or at least complex interpretations, underscoring the point that distinguishing effects of stress on performance from those on learning remains a serious issue that is not easily resolved (Maier and Jackson 1979; Minor et al. 1991; Wasserman and Miller 1997; Miller and Matzel 2000; Cahill et al. 2001).

\section{Stress and Spatial Learning}

In addition to performance effects, there are potential pitfalls when trying to interpret the significance of stress effects on learning. To cite an example, several years ago, we evaluated the effects of an inescapable stressor on subsequent performance in a spatial maze task known to require an intact hippocampal formation-the 8-arm radial maze task. The maze, designed by Olton in the 1970s (Olton and Samuelson 1976), consists of a central platform from which radiate eight arms, like the spokes of a wheel. Typically, the extremity of each arm is baited with a food 
pellet placed in a depressed circular hole. At the beginning of a trial, an animal is placed on the central platform; it then runs down various arms to consume the food pellets. A hungry rat rapidly learns that each arm contains only one pellet and avoids arms that were already explored and debaited, instead entering unexplored arms until all pellets have been consumed. To remember which arms it has entered, the animal uses salient spatial cues that surround the maze. For this reason, the maze has proved a valuable tool for the study of spatial memory and the neural substrates that mediate it.

Performance in the maze has been measured in a variety of ways. The most common measure is the number of re-entries into an arm from which the food pellet has already been removed (re-entries into the debaited arms). In our experiment, rats were exposed to the inescapable stressor of brief intermittent tail shocks, and $24 \mathrm{hr}$ later, trained on the maze task. As shown in Figure $1 \mathrm{~A}$, stressed rats committed relatively few errors during the first days of training, but more as training proceeded (Shors and Dryver 1992; Fig. 1A). In contrast, unstressed rats committed more errors initially, but fewer as training progressed. At first glance, these data might suggest that exposure to the stressful event impaired learning, and specifically, the ability to use spatial cues for navigation in a novel environment. However, on closer examination, it was clear that stressed animals knew exactly where they were, where they were going, and were using spatial cues to accomplish their goals. Generally, after being placed in the central area, the stressed animal would enter an arm, proceed to the end, eat the pellet, and remain there for some period of time. This type of behavior was associated with very few errors and a decrease in activity (Fig. 1B). With time (and increasing hunger), the animal would emerge from that arm, obtain a pellet from another arm, and return to his home arm to consume it. The animal did not randomly choose other arms to obtain pellets, but rather remembered where the pellets had been consumed initially and entered alternative arms that were not debaited. The point here is that the stressed animal was not learning impaired, even though the numbers would indicate that they were. One might even propose that the animal's performance was enhanced by the stressful event, at least to the extent that the rat was hovering in the arm that was considered safe, venturing out only to obtain food and returning to consume it in the safe region. This example simply illustrates some of the problems in inferring learning effects strictly from one or two measures in a standard laboratory task. Also, this example illustrates the futility of trying to make very general statements about the relationship between stress and learning, much less whether it is good or bad.

\section{Stress and Classical Conditioning}

The problem of distinguishing effects on performance from those on learning in animals is minimized in tasks of classical conditioning, otherwise known as Pavlovian conditioning. During this type of conditioning, an animal learns to associate a stimulus with the occurrence of another stimulus that necessarily elicits a response. In our studies, we have focused on the classically conditioned eyeblink response in the freely moving rat. In this task, an auditory conditioned stimulus (CS) is preceded by and predicts the occurrence of an unconditioned stimulus (US), which is a periorbital shock to the eyelid. The shock to the eyelid elicits a blink, which is the unconditioned response (UR). When the shock to the eyelid is repeatedly paired with and preceded by the auditory stimulus, the auditory stimulus itself comes to elicit the eyeblink. This response is the conditioned response (CR), and the number of CRs throughout training can be used as a measure of performance. Because the periorbital stimulation always elicits an eyeblink (the UR), learning the association is not dependent on the ability to elicit the basic motor response. Thus, relative to instrumental conditioning, classical conditioning can be less susceptible to the effects of an experimental manipulation such as stressor exposure on performance.

Surprisingly, there had been few attempts to examine the effects of stressful and emotional experience on performance using classical conditioning procedures (Spence and Beecroft 1954). Using such a task, we observed an effect that was quite different and indeed opposite to that of helplessness. Specifically, we observed that exposure to an acute inescapable stressor of restraint and intermittent tailshocks enhances classical eyeblink conditioning in the male rat (Shors et al. 1992; Servatius and Shors 1994; Shors and Servatius 1995, 1997; Fig. 2A). This effect is rapidly induced and persistently expressed. In other words, if the training begins immediately after stress exposure, conditioning is enhanced, just as it is if training begins a day later (Shors 2001). Exposure to the stressful event does not appear to adversely affect the motor response, as exposure to the stressor during training does not alter eyeblink responding (Beylin and Shors 1998; Shors 2001; Fig. 2B). Nor does stressor exposure induce responding to the CS when the stimuli are presented in an unpaired manner (Shors et al. 1992; Fig. 2A). Finally, exposure to the stressful event does not alter the amplitude of the UR (Servatius et al. 2001). Overall, these results suggest that exposure to the stressful event enhances learning, rather than simply enhancing the motor output or performance of the response.

This effect of acute stress on classical conditioning generalizes to some, but not all conditioned responses and stressors. For example, exposure to stressful events of brief intermittent shocks enhances classical conditioning of heart rate as well as the eyeblink

Figure $1(A)$ In this experiment, male rats were exposed to an acute stressful event, and $24 \mathrm{hr}$ later, tested on performance in the 8-arm radial maze task (Shors and Dryver 1992). The graph depicts the number of errors described as reentries into an arm that had previously been debaited. On the first few days of training, stressed rats committed fewer errors than unstressed rats, but with continued training, stressed rats committed more errors and the unstressed committed fewer. $(B)$ The second graph depicts the activity of stressed and unstressed rats, defined as the arms entered per minute. Those exposed to the stressor were less active than those not exposed to the stressor, especially during the first few days of training. Overall, the data suggest that stress impedes, but does not necessarily impair spatial learning.

\section{Learning \& Memory}

www.learnmem.org 
A

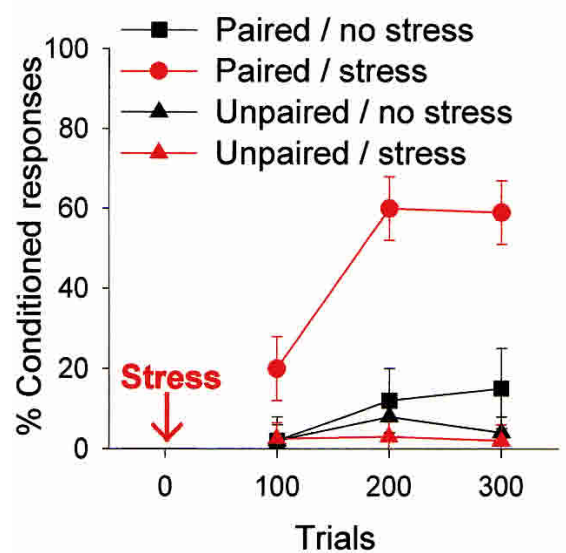

B

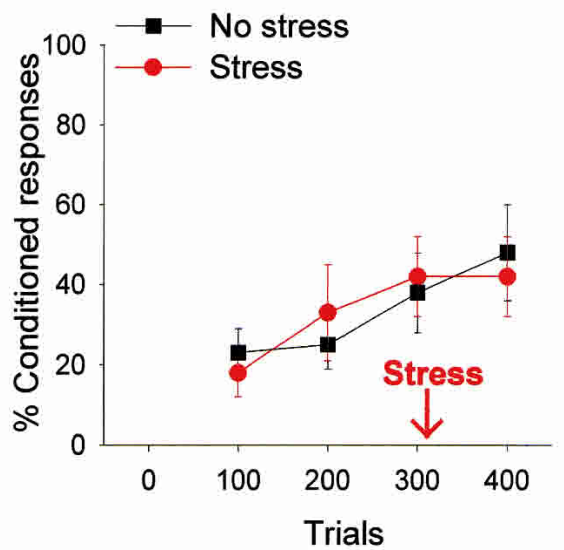

C

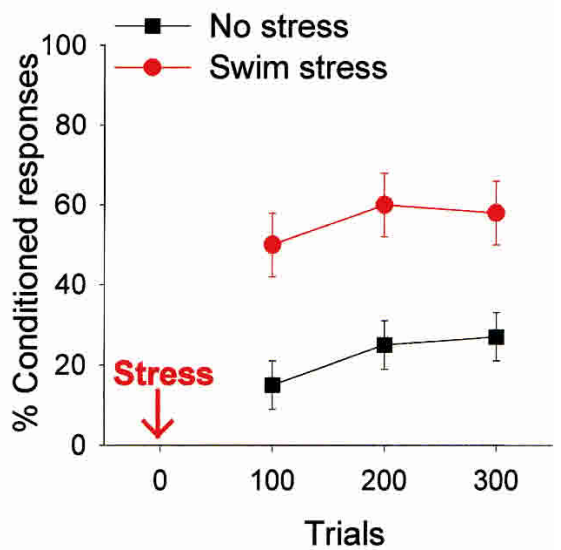

Figure $2(A)$ In this experiment, male rats were exposed to an acute stressful event of brief intermittent tail shocks, and 24 hr later, trained on the classically conditioned eyeblink response with paired stimuli consisting of a white noise conditioned stimulus (CS) and eyelid stimulation as the unconditioned stimulus (US) (Shors et al. 1992). Additional groups were exposed to the same number of stimuli presented in an explicitly unpaired manner. As shown, exposure to the stressful event greatly increased the number of learned responses to the CS, but did not induce responding to the CS when the stimuli were unpaired. $(B)$ In this experiment, male rats were exposed to the acute stressful event as the animals were acquiring the CR. In this case, exposure to the stressful event did not alter responding to the CS (Shors 2001). (C) In this experiment, male rats were exposed to an acute stressor of 20 min inescapable swimming, and trained $24 \mathrm{hr}$ later on the classically conditioned eyeblink response (Shors 2001 ). Exposure to the acute swim stress increased the number of learned responses to the CS. Overall, these data indicate that acute stressful experience enhances the ability to acquire new associations involving fine motor responses, at least in males.

response (Wilson et al. 1975). Interestingly, we have not observed enhancing effects of this type of stressor on classical fear conditioning using a decrease in movement as the measure of performance (D.A. Townsend and T.J. Shors, in prep.), although there are reports of such effects in the literature (Maier 1990). In terms of the stressor, we have reported very similar effects of stress on eyeblink conditioning in response to a brief episode (20 min) of swim stress (Shors 2001; Fig. 2C). Others have observed that water deprivation greatly enhances acquisition of the conditioned eyeblink response (Berry and Swain 1989). However, enhancement is not induced by all stressors. For example, neither restraint alone nor noise stress altered subsequent eyeblink conditioning (Shors 2001). Nevertheless, data from a number of studies now indicate that in contrast to helplessness effects, exposure to an uncontrollable stressful event can facilitate performance of a classical conditioned response-as detected in male animals.

\section{Opposite Effects of Stress on Conditioning in Males Versus Females}

If one were to conduct the same types of experiments as described, but in females rather than males, the results would be very different. In fact, the results can be quite the opposite. Exposure to the acute stressful events of either tailshocks or swim stress that enhances conditioning in males, instead, dramatically impairs conditioning in females (Wood and Shors 1998; Wood et al. 2001; Fig. 3). Thus, in response to the same environmental stimuli and experimental conditions, males and females respond in opposite directions. This effect of stress on conditioning in females, like that of males, does not appear to be directly attributable to nonspecific effects of stress on performance. Neither stress nor sex differences altered the number of spontaneous blinks or blinks in response to auditory stimuli prior to training. Neither stress nor sex differences enhanced responding to the conditioning stimuli when they were presented in an explicitly unpaired manner, nor did they induce responses during the first trial of paired training before any learning could have occurred. Although females tend to be more active than males and less activity impaired in response to stressor exposure, they were not more active $24 \mathrm{~h}$ after the stressor and at the time of training. On the basis of reports that female rats have lower thresholds for flinching and jumping in response to shock (Drury and Gold 1978), we considered the potential contribution of sexually dimorphic analgesic responses to the stressful event. However, we have found no evidence that exposure to the stressor altered pain sensitivity in females, or males for that matter (Wood and Shors 1998), or that the UR was affected by exposure to the stressor (D.A. Bangasser and T.J. Shors, in prep.). As noted previously, one cannot rule out all potential effects of stress on behaviors that

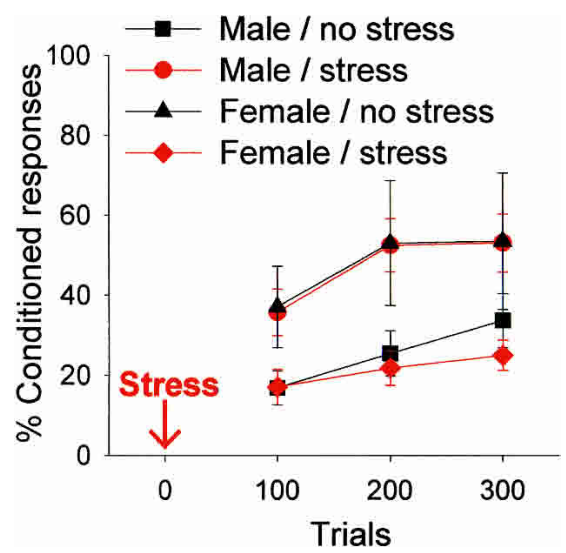

Figure 3 In this experiment, males and females were exposed to the stressful event, and $24 \mathrm{~h}$ later, trained on the classically conditioned eyeblink response using a trace paradigm that is dependent on the hippocampal formation for acquisition (Beylin et al. 2001; Wood et al. 2001). Exposure to the stressful event increased the number of learned responses in males, but reduced the number of learned responses in females, especially when they were tested in proestrus. There are also sex differences in conditioning itself, with females outperforming males under unstressed conditions. Overall, these data indicate that males and females can respond in opposite directions to the same environmental event. 
affect performance. Even so, these findings represent very different behavioral responses to stress-simply as a matter of sex.

\section{Sex Differences in Helplessness}

Perhaps such different responses to stress in females should not be so surprising. After all, for decades now, it has been known that females are particularly sensitive to stressful life events (Barnett et al. 1987). In humans, females are more susceptible to stress-related illness, including depression, generalized anxiety, and post-traumatic stress disorders (Shors 2002; Shors and Leuner 2003). In the animal literature, there are numerous indications that females are very different in their stress responsivity (Shalev and Weiner 2001; Wolf et al. 2001; Bowman et al. 2003). For learned helplessness, the pre-eminent theory regarding stress effects on behavior, it is reported that female rats do not express the phenomenon itself. That is, it has been reported that exposure to an uncontrollable stressor does not induce the impaired performance in operant conditioning that is typically observed in males (Kirk and Blampied 1985; Steenbergen et al. 1990). Overall, female rats tend to be more active than males and less likely to freeze after exposure to uncontrollable stressors such as brief tailshocks. Similarly, they learn more quickly than males if the task requires an active response such as during escape training, but learn poorly if the task requires passivity as in passive avoidance conditioning (for review, see Shors 1998). Thus, the absence of helplessness behavior in females may simply reflect their general tendency to move more than males, especially after exposure to the stressful event. Such behaviors would expose them to the environment, and thereby enhance their opportunities for learning.

In our studies, we found that the detrimental effects of stress on classical conditioning procedures are most evident during specific stages in the reproductive cycle. The rat estrous cycle encompasses a $4-5 \mathrm{~d}$ period with dramatic fluctuations in estrogen and progesterone, among other hormones. Peak estrogen levels occur just prior to ovulation and during the stage of proestrus. The effects of acute stress on classical conditioning are most evident in females that are exposed to the stressor prior to proestrus (diestrus 2) and trained subsequently in proestrus (Shors et al. 1998). It is during this stage of proestrus that females are most active. In fact, voluntary activity in a running wheel is often used to monitor stages of estrous. As discussed, it has been reported that females do not express the helplessness phenomenon. However, in these studies, the estrous cycle was not monitored. In a more recent study, Petty and colleagues did evaluate the effects of stress on escape behavior during specific stages of the cycle (Jenkins et al. 2001). Specifically, males and females were exposed to either inescapable or escapable shocks, and $1 \mathrm{~h}$ later, tested in their escape ability in a shuttle-box apparatus (to ensure that they remained in the same stage of estrus). During training, they were first exposed to a series of trials in which they had to shuttle once from one side of the chamber to the other in order to terminate the footshock (a fixed-ratio one; FR-1). Then, they were exposed to trials in which they had to shuttle back and forth to terminate the foot shock (a fixed-ratio two; FR-2). Males showed no deficit in the one-way task, but were delayed in acquiring the two-way task. In contrast, females were delayed in responding during the one-way and the two-way task when tested in diestrus 2, prior to the onset of proestrus. They were not impaired when tested during estrus. It is often asserted that the absence of a deficit on the one-way task reflects the absence of a performance deficit. If this is the case, then females are expressing a performance deficit, and perhaps not one of learning. In any case, these various data indicate that the effects of stress on conditioning depend not only on the stressor and the task, but also on the sex of the animal.

\section{Adaptive or Epiphenomenonal?}

What might be the evolutionary benefits or adaptive significance of these sex differences in learning and responses to stress? That stressful experience would enhance the ability to process and acquire new information, even associations, is easy to reconcile with theories of natural selection and survival. Seemingly, in the wild, it would be adaptive to maintain a state of heightened vigilance and attention during the period of time immediately after experiencing a life-threatening event. One could imagine that this particular response would be useful for males, as they often have to defend territory and resources under stressful conditions. The adaptive utility of the female response (that of impaired performance after stress) is more difficult to reconcile with theories of natural selection and survival, and must be considered in the context of their learning abilities under unstressed conditions. That is, even in the absence of stress manipulation, there are sex differences in performance (Shors et al. 1998; Wood et al. 2001). Simply put, females in proestrus emit more learned responses than males and females in other stages of estrus. As mentioned, proestrus is a stage in the rat cycle before ovulation when estrogen levels are high. Female behavior is quite different during this stage, with increases in exploration and decreases in food and water intake. Over their lifetime, female rats in the wild have relatively few seasons and, hence, few opportunities to become pregnant; each proestrus in season is a critical time for finding a mate, yet avoiding capture by prey. Thus, it makes intuitive sense that females would perform optimally during this time period. Presumably, other behavioral changes, such as increased activity and reduced food and water intake, are similarly adaptive. But why would females be so sensitive to stressful events during this time? One possible explanation is that exposure to a stressful and potentially life-threatening event signals to the animal that there are dangerous stimuli in the vicinity and now is not the time to explore, much less reproduce. In this scenario, the effect of stress on conditioning is not viewed so much as an impairment, but rather an amelioration of enhanced responding that would normally occur during this stage of reproductive life.

As a final consideration, it is possible that these opposite effects of stress on conditioning and performance in males versus females are epiphenomenal and only observed under limited conditions in a laboratory situation. Even so, these findings reveal very different, opposite effects in the same species to the same environmental stimulus. If nothing else, they indicate that there is no simple or even consistent relationship between stress and learning. To emphasize the point, I now present some data indicating that the effects of stress on some learning tasks can be eliminated, and even reversed, simply by exposing animals to different sex hormones in utero or upon birth.

\section{Reversing the Effects of Stress on Learning}

There is vast literature documenting that exposure to hormones early in development can alter behaviors expressed in adulthood. The majority of studies report changes in sexual behavior, such as mounting in males and lordosis, a posture that indicates sexual receptivity in females. For example, males castrated at birth will exhibit the female-typical response of lordosis when exposed to ovarian hormones during adulthood (Phoenix et al. 1959; Feder and Whalen 1965; Pfaff et al. 2002). In contrast, females that are exposed briefly to testosterone at birth do not ovulate or exhibit lordosis, but exhibit male sexual behaviors such as mounting in response to testosterone exposure. From these studies, it is proposed that the background condition for reproductive behaviors is feminine, and the presence of testosterone during early development organizes a masculine response (Jost et al. 1973; Arnold and Breedlove 1985; Becker et al. 1992).

\section{Learning \& Memory}


On the basis of this literature, we considered the possibility that the opposite effects of acute stress on conditioning in male and female rats might be altered or even reversed by manipulating sex hormones during very early development. In the first experiment, we manipulated testosterone in both sexes on the day of their birth (Fig. 4). We asked whether exposure to testosterone in females would alter their response to that of males, that is, would their learning be enhanced by exposure to stress in adulthood? We also asked whether removing testosterone from males on the day of birth would alter their behavior to that of the females, that is, would their learning now be impaired by exposure to stress? As shown in Figure 4B, learning in adult males that had been castrated at birth was still enhanced by exposure to the stressor, and thus, removal of testosterone after birth did not affect this behavior. In females, however, there was a dramatic change; they responded in the opposite direction as adults, that is, their performance was facilitated by stress (Fig. 4A). Thus, exposure to testosterone upon birth prevented the typical female response to stress and induced a male-like response. Sex differences in conditioning were also minimized by this manipulation.

Next, we considered the possibility that castration did not alter the male-typical response, because it was already established in utero (i.e., the brain had already been exposed to testosterone). This would not be unheard of, as male rats experience a substantial increase in testosterone levels on about day 17 of gestation, which drops to female levels by day 20. If exposed to a testosterone antagonist in utero, they exhibit reduced sexual behavior, preference for males, and can display lordosis responses in the presence of estrogen (Nadler 1969; Vega Matuszczyk and Larsson 1995). Therefore, we hypothesized that exposure to testosterone in utero organized the male-specific response to stress in adulthood. To test this hypothesis, pregnant female rats were injected with a testosterone antagonist (CA) for several days when testosterone levels are elevated in male pups. Foster mothers raised the offspring, and the effect of stress on associative learning was evaluated when the pups reached adulthood. Learning in the adult females was unaffected by antagonizing testosterone in utero; that is, stress still impaired their performance. However, as shown in Figure 4C, performance was not enhanced by stress in the treated males. It is noted that stress did not impair learning in these males, and thus, the manipulations during gestation did not reverse the male response, but rather, prevented a response from occurring. In summary, these data indicate that the presence of sex hormones during gestation and development organize whether and how acute stress will affect the ability to acquire new information in adulthood (Shors and Miesegeas 2002). Moreover, they indicate that the relationship between stress and learning is not absolute and can be manipulated quite dramatically by changes that occur as the brain becomes organized for future encounters with stressful life events.

\section{Existing Theories, Functions, and Multiple Memory Systems}

The relationship between stress and learning is often described theoretically in terms of an inverted- $\mathrm{U}$, and is illustrated in Figure $5 \mathrm{~A}$. At very low levels of stress (i.e., boredom or drowsiness), performance is impaired; at moderate levels of stress, performance is enhanced; but at extreme levels of stress, performance is impaired. Although some correlates of stress such as levels of glucocorticoids can relate to performance in such a manner (Roozendaal 2001), others do not. For example, the relationship between inescapable stress and classical eyeblink conditioning does not adhere to this function. Using a variety of stressors of varying intensities and qualities, we have found that after exposure to the stressor, conditioning is either enhanced or it is not. There is no observable gradation in responses; it appears to be an all-or-none phenomenon. Exposure to both the acute swim stress (20 min) and 30 intermittent tailshocks $(1 \mathrm{~mA}, 1 \mathrm{sec})$ enhanced conditioning in males, whereas exposure to $90 \mathrm{~min}$ of restraint or noise stress, both of which enhanced corticosterone levels similarly, did not (Shors and Servatius 1997; Shors 2001). Lesser amounts of stress, varying shock numbers or intensity, also did not alter conditioning. Of course, it is conceivable, perhaps probable, that if enough stress were delivered, performance in males would be impaired. However, such degrees of stimulation would be more traumatic than stressful, per se. In summary, an inverted-U-type function does not describe the effects of acute stressful experience on classical conditioning of a fine motor response such as the eyeblink.

One of the most cited functions relating stress (or arousal) to learning was posed by Yerkes and Dodson in 1908, and was thereafter known as the Yerkes-Dodson Law. This theory, like that of
A

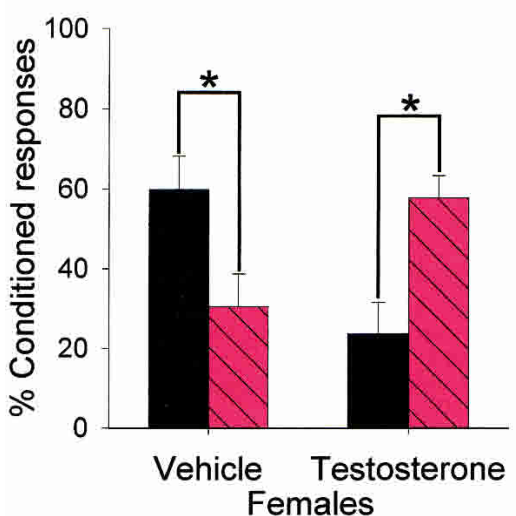

B

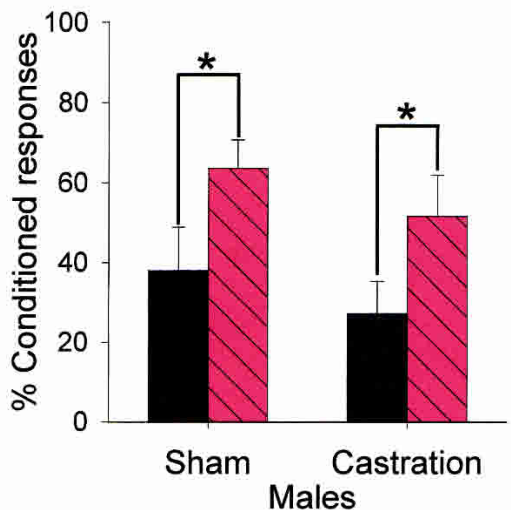

C

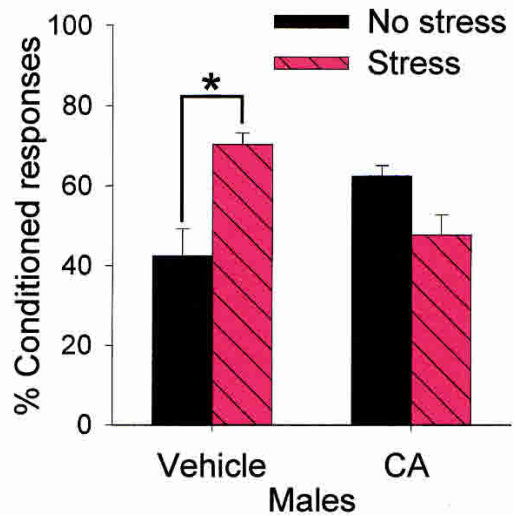

Figure 4 In this set of experiments, male and female rats were exposed to different hormonal manipulations during very early development and tested as adults in their performance during trace conditioning and their response to stressful experience (Shors and Miesegeas 2002). ( $A$ ) Females injected with one dose of vehicle on the day that they were born emitted many fewer learned responses after stress in adulthood. However, those injected with testosterone on the day that they were born emitted many more learned responses after stress in adulthood. ( $B$ ) Males castrated on the day that they were born or exposed to a sham surgery emitted more learned response after stress in adulthood. (C) However, males in which testosterone receptors were antagonized in utero did not respond to acute stress in adulthood, whereas those exposed to a vehicle in utero emitted more learned responses after stress in adulthood. Overall, these data indicate that stress effects on learning are organized very early in development and are remarkably plastic. 
A

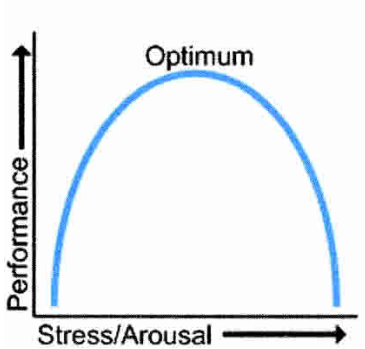

B

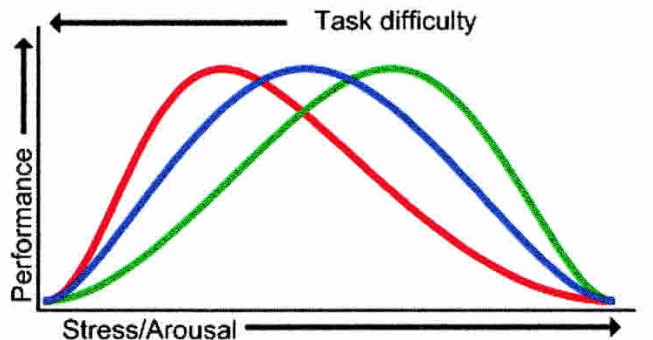

C

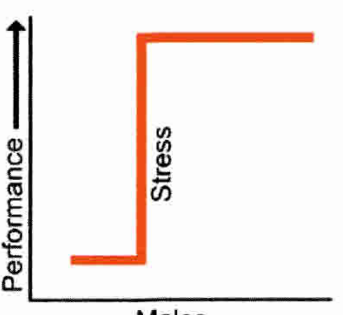

Males

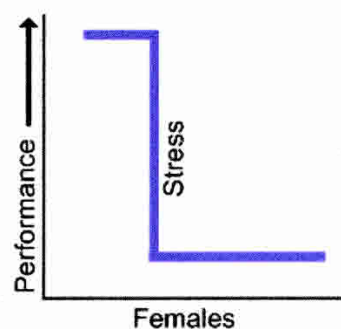

Females

Figure $5(A)$ The inverted-U shaped relationship between stress and learning is depicted with optimum performance occurring at moderate levels of stress. Poor performance would occur with low and very high levels of stress. $(B)$ The Yerkes-Dodson law is depicted with three levels of task difficulty. At low levels of task difficulty, higher levels of stress induce optimum levels of performance. At high degrees of task difficulty, lower levels of stress induce optimum levels of performance. (C) The relationship between stress and performance in males and females is depicted as a punctate response that either occurs or does not. After a threshold of stress is reached in males, performance in enhanced. After threshold is reached in females, performance is impaired.

an inverted-U, poses a relationship based on degrees of stress and performance. However, the theory is multidimensional, with an additional axis for task difficulty. As illustrated in Figure 5B, low levels of stress (or arousal) are associated with enhanced performance on more difficult tasks, whereas higher degrees of stress are associated with enhanced performance on easier ones. We have examined whether or not the effects of stress on classical eyeblink conditioning depend on task difficulty. To do that, we tested the effects of stress on two types of conditioning, one easier than the other. Delay conditioning is a relatively easy task, in which the CS (tone) and eyelid stimulus (US) stimuli overlap in time (Fig. 6A). Trace conditioning, on the other hand, is a more difficult task. During trace conditioning, the stimuli are separated in time, and thus, the animal must maintain a representation of the CS after its offset in order to associate it with the second stimulus (Fig. 6B). Most animals, including humans, require many more trials to learn the trace association than the delay one (using the same intertrial interval; Woodruff-Pak 1993; Beylin et al. 2001). In males, exposure to the acute, nonetheless intense stressor of brief intermittent tailshocks enhances delay conditioning (Fig. 6A), the easier task, as well as enhancing trace conditioning, the more difficult task (Fig. 6B). Exposure to the same stressor impairs both delay and trace conditioning in females. Thus, task difficulty, per se, does not differentiate the effects of stress on classical eyeblink conditioning.
A

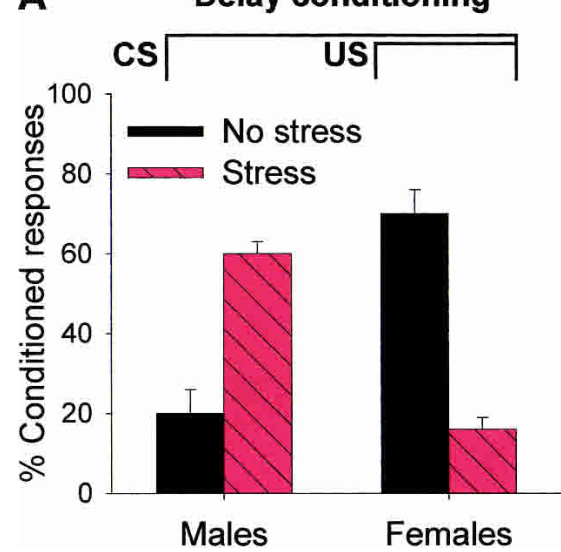

B

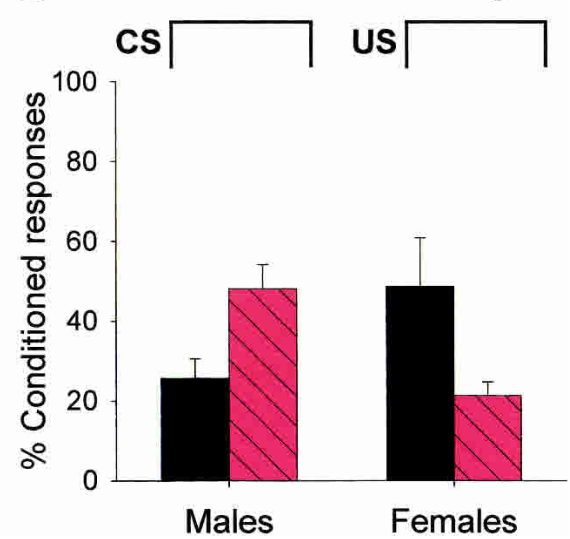

Figure 6 The opposite effects of acute stressful experience on classical eyeblink conditioning occur irrespective of task difficulty or the brain region on which the task depends. $(A)$ Acute stress enhances performance during delay conditioning in males but impairs delay conditioning in females (Wood and Shors 1998). (B) Acute stress also has opposite effects on trace conditioning in males and females, even though the task is more difficult and depends on an intact hippocampus (Beylin et al. 2001; Wood et al. 2001).

\section{Learning \& Memory \\ 142 Learning \& Memory}

It has become evident in recent years that there are a few, if not multiple memory systems in the brain (Kesner 1991; Squire and Zola 1996; Thompson and Kim 1996; Poldrack and Packard 2003). The exact details about how these systems interact are still unknown, but it has become popular to pose various phenomena on the basis of a system's reliance, or lack thereof, on the hippocampus. For example, some have posed that stress effects on learning depend on whether the animal has sufficient resources to use a hippocampal-based memory system (Diamond et al. 1996; Luine et al. 1996; Kim and Diamond 2002; see Lupien and Lepage 2001 for alternative view). Although this general idea is consistent with a number of observations (Akirav et al. 2001), it is inconsistent with those involving classical eyeblink conditioning, in which the response to stress is similar, irrespective of whether the task requires the hippocampus or not. Delay conditioning does not require the hippocampus for learning, whereas trace conditioning does (Solomon et al. 1986; Weiss et al. 1999; Beylin et al. 2001), yet both are similarly enhanced by stress in males and impaired by stress in females (Fig. 6). Thus, stressinduced effects on eyeblink conditioning are not limited to a particular type of conditioning task or brain region associated with learning that task.

There are certainly examples in which stress and performance adhere to these various functions, be they inverted, upright, or simply linear. However, the relationship between stress and classical eyeblink conditioning adheres to a step function, in which the effect of stress on conditioning is either induced or not (Fig. 5C). Depending on sex, the effect can be positive or negative, but it is expressed as a punctate response to stressful life experience.

\section{Conclusion}

To summarize, exposure to an acute stressful event has numerous effects on learned behaviors and performance. It can impede learning to escape from an aversive stimulus, alter perseverative behavior, and thereby impede performance on a spatial learning task. It can enhance or impair performance of a classically conditioned motor response, simply as a matter of sex. Moreover, its' effects on performance can be altered, and in some cases, reversed simply by manipulating hormonal milieu during very early development. What do such different responses to the same stressful 
events reveal about the true relationship between stress and learning, other than its apparent complexity? First, it is unlikely that there are one or even two defining relationships between stress and learning. Second, they are not exclusively negative. And finally, the neuronal mechanisms that mediate these differing, and in some cases, opposite responses to stress must be remarkably plastic and broad in their capabilities. Perhaps the capacity to respond in very different ways to stressful stimuli is the hallmark of a highly evolved and adaptive stress response. By appreciating, even capitalizing on its plasticity, it may be possible to more accurately describe relationships between stress and learning as well as the neuronal mechanisms that underlie them.

\section{ACKNOWLEDGMENTS}

This work was supported by National Institute of Mental Health (R01-59970), National Science Foundation (IBN0217403), and the National Alliance for Research on Schizophrenia and Depression (NARSAD). I thank Jacqueline Falduto for technical assistance.

\section{REFERENCES}

Akirav, I., Sandi, C., and Richter-Levin, G. 2001. Differential activation of hippocampus and amygdala following spatial learning under stress. Eur. J. Neurosci. 14: 719-725.

Anisman, H., deCantanzaro, D., and Remington, G. 1978. Escape performance following exposure to inescapable shock: Deficits in motor response maintenance. J. Exp. Psychol.: Anim. Behav. Process 4: $197-218$.

Arnold, A.P. and Breedlove, S.M. 1985. Organizational and activational effects of sex steroids on brain and behavior: A reanalysis. Horm. Behav. 19: 469-498.

Barnett, R.C., Biener, L., and Baruch, G.K. 1987. Gender and stress. The Free Press, New York.

Becker, J.B., Breedlove, S.M., and Crews, D. 1992. Behavioral endocrinology. MIT Press, Cambridge, MA.

Berry, S.D. and Swain, R.A. 1989. Water deprivation optimized hippocampal activity and facilitates nictitating membrane conditioning. Behav. Neurosci. 105: 202-209.

Beylin, A.V. and Shors, T.J. 1998. Stress enhances excitatory trace eyeblink conditioning and opposes acquisition of inhibitory conditioning. Behav. Neurosci. 112: 1327-1338.

Beylin, A.V., Talk, A.C., Gandhi, C.C., Wood, G.E., Matzel, L.D., and Shors, T.J. 2001. The role of the hippocampus in trace conditioning: Temporal incongruity or task difficulty? Neurobiol. Learn. Mem. 76: $447-461$.

Bowman, R.E., Beck, K.D., and Luine, V.N. 2003. Chronic stress effects on memory: Sex differences in performance and monoaminergic activity. Horm. Behav. 43: 48-59.

Cahill, L., McGaugh, J.L., and Weinberger, N.M. 2001. The neurobiology of learning and memory: Some reminders to remember. Trends Neurosci. 25: 77-78.

Diamond, D.M., Fleshner, M., Ingersol, N., and Rose, G.M. 1996. Psychological stress impairs spatial working memory:relevance to electrophysiological studies of hippocampal function. Behav. Neurosci. 110: 661-672.

Drury, R.A. and Gold, R.M. 1978. Differential effects of ovarian hormones on reactivity to electric footshock in the rat. Physiol. Behav. 20: 187-191.

Feder, H.H. and Whalen, R.E. 1965. Feminine behavior in neonatally castrated and estrogen-treated male rats. Science 147: 306-307.

Glazer, H.I. and Weiss, J.M. 1976. Long-term interference effect: An alternative to "learned helplessness". J. Exp. Psychol.: Anim. Behav. Process 2: 202-213.

Jackson, R.L., Alexander, J.H., and Maier, S.F. 1980. Learned helplessness, inactivity, and associative deficits: Effects of inescapable shock on response choice escape learning. J. Exp. Psychol.: Anim. Behav. Process 6: 1-20.

Jenkins, J.A., Williams, P., Kramer, G.L., Davis, L.L., and Petty, F. 2001. The influence of gender and the estrous cycle on learned helplessness in the rat. Biol. Psychiatry 58: 147-158.

Jost, A., Vigier, B., Prepin, J., and Perchellet, J.P. 1973. Studies on sex differentiation in mammals. Recent Prog. Horm. Res. 29: 1-41.

Kesner, R. 1991. Neurobiological views of memory. In Learning and memory (eds. J.L. Martinez Jr. and R. Kesner), pp. 399-438. Academic Press, Orlando, FL.

Kim, J.J. and Diamond, D.M. 2002. The stressed hippocampus, synaptic plasticity and lost memories. Nat. Neurosci. Rev. 3: 453-462.

Kirk, R.C. and Blampied, N.M. 1985. Activity during inescapable shock and subsequent escape avoidance learning: Female and male rats compared. New Zealand J. Psychol. 14: 9-14.

Luine, V.N., Martinez, C., Villegas, M., Margarinos, A.M., and McEwen, B.S. 1996. Restraint stress reversibly enhances spatial memory performance. Physiol. Behav. 59: 27-32.

Lupien, S.J. and Lepage, M. 2001. Stress, memory, and the hippocampus: Can't live with it, can't live without it. Behav. Brain Res. 127: 137-158.

Maier, S.F. 1990. Role of fear in mediating shuttle escape learning deficit produced by inescapable shock. J. Exp. Psychol.: Anim. Behav. Process 16: $137-149$.

Maier, S.F. and Jackson, R.L. 1979. Learned helplessness: All of us were right (and wrong): Inescapable shock has multiple effects. In Advances in learning and motivation (ed. B. Bower), pp. 155-215. Academic Press, New York.

Miller, R.R. and Matzel, L.D. 2000. Memory involves far more than 'consolidation'. Nat. Neurosci. Rev. 1: 214-216.

Minor, T.R., Jackson, R.L., and Maier, S.F. 1984. Effects of task-irrelevant cues and reinforcement delay on choice escape learning following inescapable shock: Evidence for a deficit in selective attention. $J$. Exp. Psychol.: Anim. Behav. Processes 10: 543-556.

Minor, T.R., Pelleymounter, M.A., and Maier, S.F. 1988. Uncontrollable shock, forebrain norepinephrine, and stimulus selection during choice-escape learning. Psychobiology 16: 135-145.

Minor, T.R., Dess, N.K., and Overmier, J.B. 1991. Inverting the traditional view of 'learned helplessness'. In Fear, avoidance, and phobias: A fundamental analysis (ed. M.R. Denny), pp. 87-133. Lawrence-Erlbaum Associates, Hillsdale, NJ.

Nadler, R.D. 1969. Differentiation of the capacity for male sexual behavior in the rat. Horm. Behav. 1: 53-63.

Olton D.S. and Samuelson, R.J. 1976. Remembrance of places passed: Spatial memory in rats. J. Expt. Psychol. Animal Behavior Processes 2: 97-116.

Overmier, J.B. and Seligman, M.E.P. 1967. Effects of inescapable shock on subsequent escape and avoidance learning. J. Comp. Physiol. Psychol. 63: 23-33.

Pfaff, D., Frohlich, J., and Morgan, M. 2002. Hormonal and genetic influences on arousal-sexual and otherwise. Trends Neurosci. 25: $45-50$.

Phoenix, C.H., Goy, R.W., Gerall, A.A., and Young, W.C. 1959. Organizing action of prenatally administered testosterone propionate on the tissues mediating mating behavior in the female guinea pig. Endocrinology 65: 369-382.

Poldrack, R.A. and Packard, M.G. 2003. Competition among multiple memory systems: Converging evidence from animal and human brain studies. Neuropsychologia 41: 245-251.

Roozendaal, B. 2001. Glucocorticoids and the regulation of memory consolidation. Psychoneuroendocrinology 25: 213-238.

Seligman, M.E.P. 1975. Helplessness. Freeman, San Francisco. CA.

Seligman, M.E.P. and Maier, S.F. 1967. Failure to escape traumatic shock. J. Comp. Physiol. Psychol. 74: 1-9.

Servatius, R.J. and Shors, T.J. 1994. Exposure to inescapable stress persistently facilitates associative and nonassociative learning in rats. Behav. Neurosci. 108: 1101-1106.

Servatius, R.J., Brennan, F.X., Beck, K.D., Beldowicz, D., and Coyle-DiNorcia, K. 2001. Stress facilitates acquisition of the classically conditioned eyeblink response at both long and short interstimulus intervals. Learn Motiv. 32: 178-192.

Shalev, U. and Weiner, I. 2001. Gender-dependent differences in latent inhibition following prenatal stress and corticosterone administration. Behav. Brain Res. 126: 57-63.

Sherman, A.D. and Petty, F. 1980. Neurochemical basis of the action of anti-depressants on learned helplessness. Behav. Neural. Biol. 30: $119-134$.

Shors, T.J. 1998. Stress and sex effects on associative learning: For better or for worse. The Neuroscientist 4: 353-364.

. 2001. Acute stress rapidly and persistently enhances memory formation in the male rat. Neurobiol. Learn. Mem. 75: 10-29. . 2002. Opposite effects of stressful experience on memory formation in males versus females. Dialog. Clin. Neurosci. 4: 39-47.

Shors, T.J. and Dryver, E. 1992. Stress impedes exploration and the acquisition of spatial information in the eight-arm radial maze. Psychobiology 20: 247-253.

Shors, T.J. and Leuner, B. 2003. Estrogen-mediated effects on depression and memory formation in females. J. Affective Disorders 74: 85-96.

Shors, T.J. and Miesegeas, G. 2002. Testosterone in utero and at birth determine how a stressful experience will affect memory formation in adulthood. Proc. Natl. Acad. Sci. 99: 13955-13960.

Shors, T.J. and Servatius, R.J. 1995. Stress-induced sensitization and facilitated learning require NMDA receptor activation. Neuroreport 
6: $677-680$.

1997. The contribution of stressor intensity, duration, and context to the stress-induced facilitation of associative learning. Neurobiol. Learn. Mem. 67: 92-96.

Shors, T.J., Weiss, C., and Thompson, R.F. 1992. Stress-induced facilitation of classical conditioning. Science 257: 537-539.

Shors, T.J., Lewczyk, C., Paczynski, M., Mathew, P.R., and Pickett, J. 1998. Stages of estrous mediate the stress-induced impairment of associative learning in the female rat. Neuroreport 9: 419-423.

Solomon, P.R., van der Schaaf, E.R., Thompson, R.F., and Weisz, D 1986. Hippocampus and trace conditioning of the rabbit's classically conditioned nictitating membrane response. Behav. Neurosci. 100: $729-744$.

Spence, K.W. and Beecroft, R.S. 1954. Differential conditioning and level of anxiety. I. Exp. Psychol. 48: 399-403.

Squire, L.R. and Zola, S.M. 1996. Structure and function of declarative and non-declarative memory systems. Proc. Natl. Acad. Sci. 93: 13515-13522.

Steenbergen, H.L., Heinsbroek, R.P.W., van Hest, A., and van de Poll, N.E. 1990. Sex-dependent effects of inescapable shock administration on shuttle-box escape performance and elevated plus-maze behavior. Physiol. Behav. 48: 571-576.

Thompson, R.F. and Kim, J.J. 1996. Memory systems in the brain and localization of memory. Proc. Natl. Acad. Sci. 93: 13438-13444.
Vega Matuszczyk, J. and Larsson, K. 1995. Sexual preference and feminine and masculine sexual behavior of male rats prenatally exposed to antiandrogen or antiestrogen. Horm. Behav. 29: 191-206.

Wasserman, E.A. and Miller, R.R. 1997. What's elementary about associative learning? Annu. Rev. Psychol. 48: 573-607.

Weiss, C., Bouwmeester, H., Power, J.M., and Disterhoft, J.F. 1999. Hippocampal lesions prevent trace eyeblink conditioning in the freely moving rat. Behav. Brain Res. 99: 123-132.

Wilson, L.M., Wilson, J.R., and Dicara, L.V. 1975. Facilitation of Pavlovian conditioned cardiodecelerations following preshock in immobilized rats. Physiol. Behav. 15: 653-658.

Wolf, O.T., Schommer, N.C., Hellhammer, D.H., McEwen, B.S., and Kirschbaum, C. 2001. The relationship between stress induced cortisol levels and memory differs between men and women. Psychoneuroendocrinology 26: 711-720.

Wood, G.E. and Shors, T.J. 1998. Stress facilitates classical conditioning in males but impairs conditioning in females through activational influences of ovarian hormones. Proc. Natl. Acad. Sci. 95: 4066-4071.

Wood, G.E., Beylin, A.V., and Shors, T.J. 2001. The contribution of adrenal and reproductive hormones to the opposing effects of stress on trace conditioning in males versus females. Behav. Neurosci. 115: $175-187$.

Woodruff-Pak, D.S. 1993. Eyeblink classical conditioning in H.M: Delay and trace paradigms. Behav. Neurosci. 107: 911-925.

\section{Learning \& Memory}




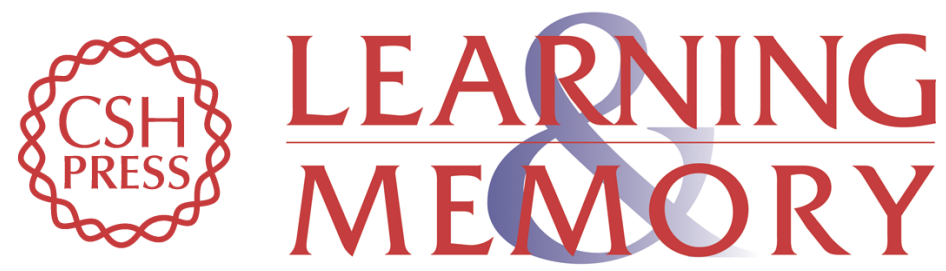

\section{Learning During Stressful Times}

Tracey J. Shors

Learn. Mem. 2004, 11:

Access the most recent version at doi:10.1101//m.66604

References This article cites 48 articles, 6 of which can be accessed free at: http://learnmem.cshlp.org/content/11/2/137.full.html\#ref-list-1

\section{License}

Email Alerting

Receive free email alerts when new articles cite this article - sign up in the box at the top Service right corner of the article or click here. 